\title{
Risk factor compositions of nonalcoholic fatty liver disease change with body mass index in males and females
}

\author{
Long Wang ${ }^{1, *}$, Jinghui Guo ${ }^{1, *}$ and Jianping Lu² \\ ${ }^{1}$ Department of Gastroenterology, Shanghai Jiao Tong University Affiliated Sixth People's Hospital, Shanghai, China \\ ${ }^{2}$ Health Examination Center, Shanghai Jiao Tong University Affiliated Sixth People's Hospital, Shanghai, China \\ * These authors have contributed equally to this work \\ Correspondence to: Long Wang, email: wanglong@medmail.com.cn \\ Keywords: nonalcoholic fatty liver disease; body mass index; gender difference; risk factor compositions; nonalcoholic steato- \\ hepatitis; Pathology Section \\ Received: April 06, 2016 \\ Accepted: May 20, 2016 \\ Published: May 30, 2016
}

\section{ABSTRACT}

Nonalcoholic fatty liver disease (NAFLD) is highly prevalent and correlated with obesity. To evaluate the role of body mass index (BMI) and gender difference in NAFLD, 8817 general adult subjects underwent physical examinations and were divided into four groups: underweight, normal, overweight and obese. The risk factor compositions for NAFLD were evaluated in each group by gender. The percentage of subjects with NAFLD increased sharply from $0.4 \%$ in the underweight group up to $81.9 \%$ in the obese group. BMI stratification showed distinct risk factor compositions associated with NAFLD in males and females according to BMI and improved the performance of NAFLD prediction models in each group. Triglycerides (TG), alanine transaminase (ALT), aspartate transaminase (AST), and uric acid were steady risk factors for NAFLD in males. Total cholesterol (TC), TG, low-density lipoprotein cholesterol (LDL-C), ALT, and uric acid were steady risk factors for NAFLD in females. TG, ALT and uric acid were common risk factors in both genders with high performance for NAFLD discrimination. Our data provide gender- and BMI-specific risk factor compositions that will facilitate individualised treatment and benefit NAFLD control and prevention.

\section{INTRODUCTION}

Fatty liver refers to hepatic steatosis accounting for more than $5-10 \%$ of the total weight of the liver or macrosteatosis of the same extent in histopathology [1]. Excessive consumption of alcohol (females $>20 \mathrm{~g} / \mathrm{d}$, males $>30 \mathrm{~g} / \mathrm{d}$ ) leads to alcoholic fatty liver [2]. While nonalcoholic fatty liver disease (NAFLD) is a spectrum of hepatic disorders, ranging from simple or bland fatty liver to nonalcoholic steato-hepatitis (NASH), which is now more common than alcoholic liver disease owing to the rapid rise in the prevalence of obesity [1-3].

NAFLD was first described in 1980 and is divided into the histological categories of nonalcoholic fatty liver and NASH [4]. Up to $75 \%$ of individuals with NAFLD are patients with isolated hepatic steatosis or with steatosis concomitant of mild nonspecific inflammation; up to $25 \%$ of individuals with NAFLD also have NASH [4-7]. Stages of isolated hepatic steatosis, steatosis with mild lobular inflammation and NASH can evolve as each other and patients with NASH have a high risk of developing liver fibrosis and liver cirrhosis [1-7]. Diabetes, insulin resistance, hypertension, weight gain and increasing alanine aminotransferase (ALT) and aspartate aminotransferase (AST) are known risk factors for disease progression from NASH to liver fibrosis [4]. Although less than $4 \%$ of individuals with nonalcoholic fatty liver will progress to cirrhosis directly, up to $20 \%$ of subjects with NASH are expected to progress to liver cirrhosis [4]. Thus the impact of NAFLD on liver disease is receiving increasing attention.

NAFLD, the hepatic manifestation of the metabolic syndrome, is the most common liver disease worldwide with prevalence estimates ranging from $25 \%$ to $45 \%[8,9]$. The proportion of NAFLD among chronic liver diseases rose from $47 \%$ to $75 \%$ between 1988 and 2008 according to data from the US National Health and Nutrition Examination Survey [10]. It is worth noting that the known risk factors for NASH - obesity, visceral obesity, type 2 
diabetes, insulin resistance and arterial hypertension-are increasing in parallel with that of NAFLD from $21.74 \%$ to $33.22 \%, 35.18 \%$ to $51.43 \%, 5.55 \%$ to $9.11 \%, 23.29 \%$ to $35.00 \%$ and $22.68 \%$ to $34.08 \%$, respectively [11]. These risk factors together with NAFLD not only constitute the leading protagonists of metabolic diseases but also have reciprocal causation and act as intermediary exacerbating factors for various diseases, including cardiovascular disease [12-14].

The risk factors for NAFLD are divided into genetics, metabolic changes, hormone changes, inflammation, diet, physical activity and the gut microbiota [15]. Encouraging progress has been made in noninvasive evaluation of NAFLD, including steatosis grading, NASH diagnosis, and liver fibrosis staging [1622]. Studies on risk factor have resulted in the formulation of NAFLD prediction algorithms, which include various parameters such as BMI, TC, TG, glucose, age, gender, waist circumference, $\gamma$-glutamyltranspeptidase, metabolic syndrome, type 2 diabetes, fasting insulin level, fasting AST level, and the AST/ALT ratio [16-20]. However, these prediction strategies have not significantly improved the clinical diagnosis of NAFLD, because there is no evidence that the performance of these algorithms exceeds that of the clinical, laboratory, and imaging evaluations that are routinely performed in patients with suspected NAFLD [21]. Thus, improvement of NAFLD prediction remains essential. Obesity is a major risk factor for NAFLD; indeed, obesity shares many risk factors with NAFLD [22]. Therefore, the confounding factors of obesity and NAFLD must be controlled for to identify NAFLD-specific risk factors. Unfortunately, of the above representative prediction algorithms [16-20], only four of five studies included obesity indexes. However, in studies that evaluated BMI and/or waist circumference [16-18,20], no stratification of obesity was performed to distinguish the impact of obesity on NAFLD and its risk factors. In addition, age, gender, and ethnicity are associated with the prevalence of NAFLD [9], but only one [16] and two $[16,18]$ of the five above studies included age and gender as variables in their predictive equations. Thus, much important information might be lost when using these algorithms. Recent studies have focused mainly on NASH instead of NAFLD [4,6], and the incidence of NAFLD has been investigated in only one study [9]. Therefore, further studies are needed to assess the incidence of NAFLD according to age, ethnicity, and geographic location.

In this study, to evaluate the role of BMI and gender in NAFLD, 8817 general Chinese subjects living in Shanghai were divided into underweight, normal weight, overweight and obese groups. The patterns of association between age, hypertension, fasting glucose, serum lipid profile, serum metabolic parameters, and NAFLD were evaluated and compared in each group according to gender.

\section{RESULTS}

\section{The distribution tendency of parameters in groups classified by BMI}

In total, 8817 subjects were enrolled in this analysis. The numbers of subjects in the underweight $(\mathrm{BMI}<18.5)$, normal $(18.5 \leq \mathrm{BMI} \leq 23.9)$, overweight $(24.0 \leq \mathrm{BMI} \leq$ $27.9)$ and obese $(\mathrm{BMI} \geq 28.0)$ groups were $445(5.0 \%)$, $4899(55.6 \%), 2801(31.8 \%)$ and $672(7.6 \%)$, respectively (Table 1). The percentages of subjects with NAFLD increased from $0.4 \%$ in the underweight group to $82.4 \%$ in the obese group. The percentages of males and females with NAFLD also increased sharply with increasing BMI. There was no significant difference in the percentages of subjects with NAFLD between males and females in the underweight, overweight and obese groups. However, the percentage of males with NAFLD was significantly higher than that of females in the normal body weight group $(14.4 \%$ vs. $11.9 \%, p=0.0156)$. The BMI, age, male ratio, systolic pressure, diastolic pressure, serum levels of TC, TG, LDL-C, glucose, ALT, AST, urea nitrogen, uric acid and creatinine were higher in groups with higher BMI. In contrast, the serum HDL-C level declined gradually with increasing BMI.

\section{Risk factors associated with NAFLD without BMI stratification}

To learn the risk factors associated with NAFLD in multiple logistic regression analysis ignoring BMI stratification, the general population was categorised by gender. The male and female groups comprised 3834 $(43.5 \%)$ and $4983(56.5 \%)$ subjects, respectively. For binary logistic regression analysis, NAFLD was used as the dependent variable, whereas BMI, age, systolic pressure, diastolic pressure, TC, TG, HDL-C, LDL-C, glucose, ALT, AST, urea nitrogen, uric acid, and creatinine were used as independent variables. All continuous variables were introduced into the starting model using actual values. As shown in Table 2, BMI, age, diastolic pressure, TG, LDL-C, glucose, ALT, AST, uric acid and creatinine are independent risk factors for NAFLD in both males and females. TC is an independent risk factor for females but not males (Table 2). Systolic pressure displayed a tendency to be an independent risk factor for males. HDL-C and urea nitrogen were not independent risk factors for either males or females.

Almost all existing reports had ignored the BMI stratification, their analytical logic was similar to what we done above. Because NAFLD was rarely observed in subjects with BMI $<18.5$ and the proportion of subjects with NAFLD increased sharply from the underweight group to the obese group, we then stratified the population 
Table 1: The distribution tendency of parameters in groups classified by BMI.

\begin{tabular}{|c|c|c|c|c|c|}
\hline \multirow{2}{*}{ Indexes } & $\mathrm{BMI}<18.5$ & $18.5<$ BMI $<23.9$ & $24.0<$ BMI $<27.9$ & $\mathrm{BMI}>28.0$ & \multirow{2}{*}{$\boldsymbol{P}$} \\
\hline & $(N=445,5.0 \%)$ & $(N=4899,55.6 \%)$ & $(N=2801,31.8 \%)$ & $(N=672,7.6 \%)$ & \\
\hline $\mathrm{BMI}\left(\mathrm{kg} / \mathrm{m}^{2}\right)$ & $17.8(17.2-18.2)$ & $21.6(20.2-22.8)$ & $25.5(24.7-26.4)$ & $29.3(28.5-30.6)$ & $<0.001$ \\
\hline Age, yrs & $32(26-48)$ & $43(32-58)$ & $51(38-61)$ & $51(37-63)$ & $<0.001$ \\
\hline Males, N (\%) & $72(16.2 \%)$ & $1628(33.2 \%)$ & $1699(60.7 \%)$ & $435(64.7 \%)$ & $<0.001$ \\
\hline Systolic pressure $(\mathrm{mmHg})$ & $117.1+15.9$ & $124.5+17.4$ & $134.4+17.9$ & $143.0+19.3$ & $<0.001$ \\
\hline Diastolic pressure (mmHg) & $70.5+9.6$ & $75.5+11.0$ & $81.8+11.2$ & $86.3+11.7$ & $<0.001$ \\
\hline $\mathrm{TC}(\mathrm{mM})$ & $4.6+0.9$ & $4.8+0.9$ & $5.1+1.0$ & $5.2+1.0$ & $<0.001$ \\
\hline $\mathrm{TG}(\mathrm{mM})$ & $0.8+0.3$ & $1.11+0.87$ & $1.66+1.35$ & $2.0+1.5$ & $<0.001$ \\
\hline HDL-C (mM) & $1.8+0.4$ & $1.6+0.4$ & $1.39+0.33$ & $1.30+0.32$ & $<0.001$ \\
\hline LDL-C (mM) & $2.5+0.7$ & $2.8+0.8$ & $3.12+0.81$ & $3.25+0.80$ & $<0.001$ \\
\hline Glucose (mM) & $5.0+1.2$ & $5.2+1.1$ & $5.6+1.3$ & $5.8+1.6$ & $<0.001$ \\
\hline $\operatorname{ALT}(\mathrm{U} / \mathrm{L})$ & $13.4+8.3$ & $16.1+12.3$ & $23.4+18.1$ & $31.9+29.4$ & $<0.001$ \\
\hline $\operatorname{AST}(\mathrm{U} / \mathrm{L})$ & $19.8+5.9$ & $20.3+8.0$ & $22.9+10.8$ & $25.8+13.4$ & $<0.001$ \\
\hline Urea nitrogen $(\mathrm{mM})$ & $4.6+1.4$ & $4.8+1.2$ & $5.1+1.3$ & $5.2+1.4$ & $<0.001$ \\
\hline Uric acid $(\mu \mathrm{M})$ & $252.7+61.5$ & $284.8+69.9$ & $336.7+75.9$ & $370.1+82.2$ & $<0.001$ \\
\hline Creatinine $(\mu \mathrm{M})$ & $58.6+13.0$ & $62.7+14.9$ & $69.8+16.0$ & $71.3+17.2$ & $<0.001$ \\
\hline NAFLD, N (\%) & $2(0.4 \%)$ & $624(12.7 \%)$ & $1379(49.2 \%)$ & $554(82.4 \%)$ & $<0.001$ \\
\hline Males with NAFLD, N (\%) & $0(0.0 \%)$ & $234(14.4 \%)^{*}$ & $830(48.9 \%)$ & $360(82.8 \%)$ & $<0.001$ \\
\hline Females with NAFLD, N (\%) & $2(0.5 \%)$ & $390(11.9 \%)$ & $549(49.8 \%)$ & $194(81.9 \%)$ & $<0.001$ \\
\hline
\end{tabular}

Normally distributed data are presented as the means \pm standard deviation (SD); skewed data are presented as the medians (interquartile range); and categorical data are presented as the numbers (percentage). Differences among groups were examined using Kruskal-Wallis H test, one-way ANOVA, $\chi 2$ tests according to the data distribution tendency. Abbreviations: BMI, body mass index; TC, total cholesterol; TG, triglycerides; HDL-C, high-density lipoprotein cholesterol; LDL-C, lowdensity lipoprotein cholesterol; ALT, alanine transaminase; AST, glutamic-oxalacetic transaminase; NAFL, nonalcoholic fatty liver. According to the categories recommended by the Chinese government, BMI $<18.5$ refers to underweight; $18.5<$ BMI $<23.9$ refers to healthy weight; $24.0<\mathrm{BMI}<27.9$ refers to overweight; $\mathrm{BMI}>28.0$ refers to obese. ${ }^{*} P=0.0156$.

into normal body weight, overweight, and obese groups to assess the impact of BMI on NAFLD and its risk factors.

\section{Risk factors associated with NAFLD in subjects with normal body weight}

To identify the risk factors associated with NAFLD in those with a normal body weight $(18.5<$ BMI $<23.9)$, a binary logistic regression analysis was performed. Age and the serum levels of TG, LDL-C, glucose, ALT and uric acid were found to be positively associated with NAFLD in both males and females (Table 3). Serum levels of TC and AST were negatively associated with NAFLD in both males and females (Table 3). Although the percentage of females with NAFLD was significantly lower than that of males with a normal body weight (Table 1), three further risk factors - BMI, diastolic pressure, and serum creatinine level - were independent risk factors for NAFLD only in females (Table 3). Systolic blood pressure, HDL-C, and urea nitrogen levels were not independent risk factors for NAFLD in males or females (Table 3 ).
Compared to the above analysis without BMI stratification (Table 2), in subjects with normal body weight, age, TG, LDL-C, glucose, ALT, AST, and uric acid were consistent risk factors in both males and females. BMI, diastolic blood pressure, and serum creatinine levels were independent risk factors for NAFLD only in females; systolic pressure was a risk factor for neither males nor females. Taken together, our data suggest that BMI stratification changes the NAFLD risk factor compositions for both males and females.

\section{Risk factors associated with NAFLD in overweight subjects}

In overweight subjects, BMI and levels of $\mathrm{TG}$, LDL-C, glucose, ALT, AST, uric acid, and creatinine were independent risk factors for both males and females; age and TC were female-specific risk factors (Table 4). Compared to subjects with normal body weight, BMI, diastolic pressure and levels of HDL-C and creatinine emerged as independent risk factors for males; age and 
Table 2: Risk factors associated with NAFLD in multiple logistic regression analysis ignoring BMI stratification.

\begin{tabular}{|c|c|c|c|c|}
\hline \multirow{2}{*}{ Risk factor } & \multicolumn{2}{|c|}{ Males $(N=3834,43.5 \%)$} & \multicolumn{2}{|c|}{ Females $(N=4983,56.5 \%)$} \\
\hline & OR $(95 \%$ CI $)$ & $P$ & OR (95\% CI) & $\boldsymbol{P}$ \\
\hline $\mathrm{BMI}\left(\mathrm{kg} / \mathrm{m}^{2}\right)$ & $1.44(1.38 \sim 1.50)$ & $<0.001$ & $1.42(1.37 \sim 1.48)$ & $<0.001$ \\
\hline Age, yrs & $1.01(1.00 \sim 1.02)$ & 0.003 & $1.03(1.02 \sim 1.040$ & $<0.001$ \\
\hline Systolic pressure (mmHg) & $0.99(0.99 \sim 1.00)$ & 0.059 & 1 & 1 \\
\hline Diastolic pressure (mmHg) & $1.02(1.01 \sim 1.03)$ & 0.001 & $1.02(1.01 \sim 1.03)$ & 0.002 \\
\hline $\mathrm{TC}(\mathrm{mM})$ & 1 & 1 & $0.40(0.32 \sim 0.52)$ & $<0.001$ \\
\hline $\mathrm{TG}(\mathrm{mM})$ & $1.16(1.08 \sim 1.25)$ & $<0.001$ & $2.01(1.75 \sim 2.29)$ & $<0.001$ \\
\hline HDL-C (mM) & 1 & 1 & 1 & 1 \\
\hline LDL-C (mM) & $1.38(1.23 \sim 1.55)$ & $<0.001$ & $3.14(2.34 \sim 4.22)$ & $<0.001$ \\
\hline Glucose (mM) & $1.18(1.11 \sim 1.26)$ & $<0.001$ & $1.17(1.08 \sim 1.27)$ & $<0.001$ \\
\hline $\operatorname{ALT}(\mathrm{U} / \mathrm{L})$ & $1.06(1.05 \sim 1.07)$ & $<0.001$ & $1.06(1.04 \sim 1.07)$ & $<0.001$ \\
\hline $\operatorname{AST}(\mathrm{U} / \mathrm{L})$ & $0.95(0.94 \sim 0.97)$ & $<0.001$ & $0.95(0.93 \sim 0.97)$ & $<0.001$ \\
\hline Usea nitrogen $(\mathrm{mM})$ & 1 & 1 & 1 & 1 \\
\hline Uric acid $(\mu \mathrm{M})$ & $1.01(1.00 \sim 1.01)$ & $<0.001$ & $1.01(1.00 \sim 1.01)$ & $<0.001$ \\
\hline Creatinine $(\mu \mathrm{M})$ & $0.99(0.98 \sim 0.99)$ & $<0.001$ & $0.98(0.97 \sim 0.99)$ & $<0.001$ \\
\hline
\end{tabular}

OR, odds ratio; CI, confidence interval. For abbreviations, see Table 1. ORs for continuous variables $=$ OR for an increase of 1 unit.

TC level lost the independent association with NAFLD in males. The risk factor panel of females was relatively stable, with the exception of diastolic pressure, which lost the independent association with NAFLD. Compared to the above analysis without BMI stratification (Table 2), in overweight subjects, BMI, TG, LDL-C, glucose, ALT, AST, uric acid, and creatinine were consistent risk factors in both males and females. The serum TC level was a consistent risk factor negatively associated with NAFLD only in females. Age and diastolic pressure were risk factors only for females and males, respectively. Similar to subjects with a normal body weight, systolic pressure was a risk factor for neither males nor females. Therefore, our data verify that BMI stratification affects the NAFLD risk factor compositions.

\section{Risk factors associated with NAFLD in obese subjects}

TG, ALT and uric acid levels were independent risk factors associated with NAFLD in both males and females with a BMI > 28.0. Compared to subjects who were overweight, the diastolic pressure and the levels of HDL-C, LDL-C and glucose were not associated with NAFLD in males (Tables 5). The BMI, age, levels of glucose, AST and creatinine were not associated with NAFLD in females, whist HDL-C levels emerged as an independent risk factor in females. Compared to the analysis without BMI stratification (Table 2), in obese subjects, only TG, ALT, and uric acid were consistent risk factors in both males and female. Serum TC level was a consistent risk factor negatively associated with NAFLD only in females. However, BMI, AST, and creatinine were risk factors only in males; age, systolic blood pressure, diastolic pressure, LDL-C, and glucose lost their independent association with NAFLD in males. Interestingly, BMI, age, systolic blood pressure, diastolic blood pressure, glucose, AST, and creatinine lost their independent association with NAFLD in females. In conclusion, BMI dramatically alters the NAFLD risk factor compositions, and risk factor compositions differ according to BMI stratification.

\section{The AUCs of each NAFLD prediction model, steady risk factors and integrated steady risk factors}

The above data show that the risk factor compositions in males and females are considerably affected by BMI. While the levels of TG, ALT, AST and uric acid were steady risk factors in males with any BMI; the levels of TC, TG, LDL-C, ALT and uric acid were steady risk factors in females with any BMI (Table 6). The levels of TG, ALT and uric acid were steady risk factors in both males and females with any BMI (Table 6 , bold labelled). Although the diastolic blood pressure was specifically associated with healthy weight females and overweight males, our data suggest that NAFLD is the result of multiple factors, and no single factor is responsible for its pathogenesis solely. To further show 


\begin{tabular}{l} 
Table 3: Risk factors associated with NAFLD in multiple logistic regression analysis in subjects with 18.5 < \\
BMI < 23.9. \\
\hline \multirow{2}{*}{ Risk factor }
\end{tabular}

\begin{tabular}{|c|c|c|c|c|}
\hline \multirow{2}{*}{ Risk factor } & \multicolumn{2}{|c|}{ Males $(\mathrm{N}=1699)$} & \multicolumn{2}{|c|}{ Females $(\mathrm{N}=1102)$} \\
\hline & OR $(95 \% \mathrm{CI})$ & $P$ & OR $(95 \% \mathrm{CI})$ & $P$ \\
\hline BMI $\left(\mathrm{kg} / \mathrm{m}^{2}\right)$ & $1.38(1.24-1.53)$ & $<0.001$ & $1.47(1.29-1.68)$ & $<0.001$ \\
\hline Age, yrs & 1 & 1 & $1.03(1.01-1.04)$ & $<0.001$ \\
\hline Systolic pressure $(\mathrm{mmHg})$ & 1 & 1 & 1 & 1 \\
\hline Diastolic pressure $(\mathrm{mmHg})$ & $1.02(1.00-1.03)$ & 0.007 & 1 & 1 \\
\hline $\mathrm{TC}(\mathrm{mM})$ & 1 & 1 & $0.36(0.25-0.53)$ & $<0.001$ \\
\hline TG (mM) & $1.10(1.01-1.20)$ & 0.035 & $1.91(1.55-2.36)$ & $<0.001$ \\
\hline HDL-C (mM) & $0.49(0.32-0.78)$ & 0.002 & 1 & 1 \\
\hline LDL-C (mM) & $1.33(1.14-1.55)$ & $<0.001$ & $3.09(1.96-4.86)$ & $<0.001$ \\
\hline Glucose (mM) & $1.20(1.10-1.31)$ & $<0.001$ & $1.16(1.01-1.34)$ & 0.042 \\
\hline $\operatorname{ALT}(\mathrm{U} / \mathrm{L})$ & $1.05(1.04-1.07)$ & $<0.001$ & $1.06(1.04-1.09)$ & $<0.001$ \\
\hline $\mathrm{AST}(\mathrm{U} / \mathrm{L})$ & $0.96(0.94-0.98)$ & $<0.001$ & $0.96(0.93-0.99)$ & 0.006 \\
\hline Urea nitrogen $(\mathrm{mM})$ & 1 & 1 & 1 & 1 \\
\hline Uric acid $(\mu \mathrm{M})$ & $1.01(1.00-1.01)$ & $<0.001$ & $1.01(1.00-1.01)$ & $<0.001$ \\
\hline Creatinine $(\mu \mathrm{M})$ & $0.99(0.98-0.99)$ & $<0.001$ & $0.96(0.95-0.98)$ & $<0.001$ \\
\hline \multicolumn{5}{|c|}{$\begin{array}{l}\text { OR, odds ratio; } \mathrm{Cl} \text {, confidence interval. For abbreviations, see Table } 1 . \text { ORs for continuous variables }=\text { OR for an increase } \\
\text { of } 1 \text { unit. }\end{array}$} \\
\hline
\end{tabular}




\begin{tabular}{|c|c|c|c|c|}
\hline \multirow{2}{*}{ Risk factor } & \multicolumn{2}{|c|}{ Males $(N=435)$} & \multicolumn{2}{|c|}{ Females $(\mathbf{N}=237)$} \\
\hline & OR $(95 \%$ CI) & $P$ & OR $(95 \%$ CI) & $P$ \\
\hline BMI $\left(\mathrm{kg} / \mathrm{m}^{2}\right)$ & $1.23(1.01-1.49)$ & 0.039 & 1 & 1 \\
\hline Age, yrs & 1 & 1 & 1 & 1 \\
\hline Systolic pressure $(\mathrm{mmHg})$ & 1 & 1 & 1 & 1 \\
\hline Diastolic pressure $(\mathrm{mmHg})$ & l & l & 1 & l \\
\hline $\mathrm{TC}(\mathrm{mM})$ & 1 & 1 & $0.11(0.03-0.45)$ & 0.002 \\
\hline $\mathrm{TG}(\mathrm{mM})$ & $1.58(1.13-2.21)$ & 0.007 & $2.83(1.29-6.18)$ & 0.009 \\
\hline HDL-C (mM) & 1 & 1 & $7.43(1.33-41.56)$ & 0.023 \\
\hline LDL-C (mM) & 1 & 1 & $15.16(3.15-73.00)$ & 0.001 \\
\hline Glucose (mM) & l & l & 1 & l \\
\hline ALT (U/L) & $1.05(1.02-1.09)$ & 0.001 & $1.10(1.03-1.17)$ & 0.003 \\
\hline AST (U/L) & $0.96(0.93-0.99)$ & 0.21 & 1 & 1 \\
\hline Urea nitrogen $(\mathrm{mM})$ & 1 & 1 & 1 & 1 \\
\hline Uric acid $(\mu \mathrm{M})$ & $1.00(1.00-1.01)$ & 0.44 & $1.01(1.00-1.01)$ & 0.032 \\
\hline Creatinine $(\mu \mathrm{M})$ & $0.97(0.95-0.99)$ & 0.016 & l & l \\
\hline
\end{tabular}

the importance of BMI stratification, the performance of each NAFLD prediction model, steady risk factor and integrated steady risk factor, was calculated according to gender. Of the steady risk factors, TG showed the highest AUCs both in males (0.74) and females (0.80); the AUCs of the integrated steady risk factors for males (TG, ALT, AST, and uric acid) and females (TC, TG, LDL-C, ALT, and uric acid) were $0.79(0.78-0.81)$ and $0.84(0.83-0.85)$, respectively (Table 6). As expected, models specific to the various BMI stratifications showed improved AUCs. The AUCs of models for males (variables: age, TC, TG, LDL-C, glucose, ALT, AST, and uric acid) and females (variables: BMI, age, diastolic pressure, TC, TG, LDL-C, glucose, ALT, AST, uric acid, and creatinine) of a healthy weight were $0.86(0.84-0.87)$ and $0.90(0.89-0.91)$, respectively (Table 6). The AUCs of models for overweight males (variables: BMI, diastolic pressure, TG, HDL-C, LDL-C, glucose, ALT, AST, uric acid, and creatinine) and females (variables: BMI, age, TC, TG, LDL-C, glucose, ALT, AST, uric acid, and creatinine) were $0.86(0.85-0.87)$ and $0.90(0.89-0.91)$, respectively (Table 6). The AUCs declined in obese subjects, those for males (variables: BMI, TG, ALT, AST, uric acid, and creatinine) and females (variables: TC, TG, HDL-C, LDL-C, ALT, and uric acid) being $0.85(0.83-0.86)$ and $0.81(0.79-0.82)$, respectively (Table 6). The cut-off values of each prediction equation for male and female subjects who were healthy weight, overweight, or obese were 0.151 (sensitivity: 0.821, specificity: 0.817) and 0.126 (sensitivity: 0.856, specificity: 0.843 ), 0.472 (sensitivity: 0.816 , specificity: 0.812 ) and 0.466 (sensitivity: 0.842 , specificity: 0.837 ), and 0.798 (sensitivity: 0.807 , specificity: 0.802 ) and 0.807 (sensitivity: 0.808 , specificity: 0.801 ), respectively. In conclusion, BMI stratification improved the performance of the NAFLD prediction models.

\section{DISCUSSION}

Together with the advancements in modern biomedical technologies and the awareness of the harm caused by NAFLD, research has progressed from risk factors to exploring a strategy to predict NAFLD and NASH $[4,23]$. Much effort has focused on identifying biomarkers to predict NASH or NAFLD; however, these strategies have rarely benefited clinical practice in terms of NAFLD diagnosis or discrimination of the pathological development of NAFLD [23]. The performance of current noninvasive evaluations of NAFLD varies among patients, aetiologies, and disease stages [23]. Ultrasonography is a simple and effective tool for steatosis screening, and liver biopsy is the gold standard for diagnosis of NAFLD in clinical practice $[21,26]$.

Many factors have been reported to be associated with NAFLD or NAFLD-related pathological processes, such as inflammation and NASH, including tumour necrosis factor- $\alpha$ [23], interleukin-6 [24], linoleic acid [25], miR-122 [26], miR-34a [26], and haptoglobin, apolipoprotein A1, tissue inhibitor of metalloproteinase 1 and chitinase-3-like protein $1[21,23]$. However, these factors represented only the general pathophysiological processes of inflammation, repair, remodelling and fibrosis. Distinguishing the pathophysiological processes 


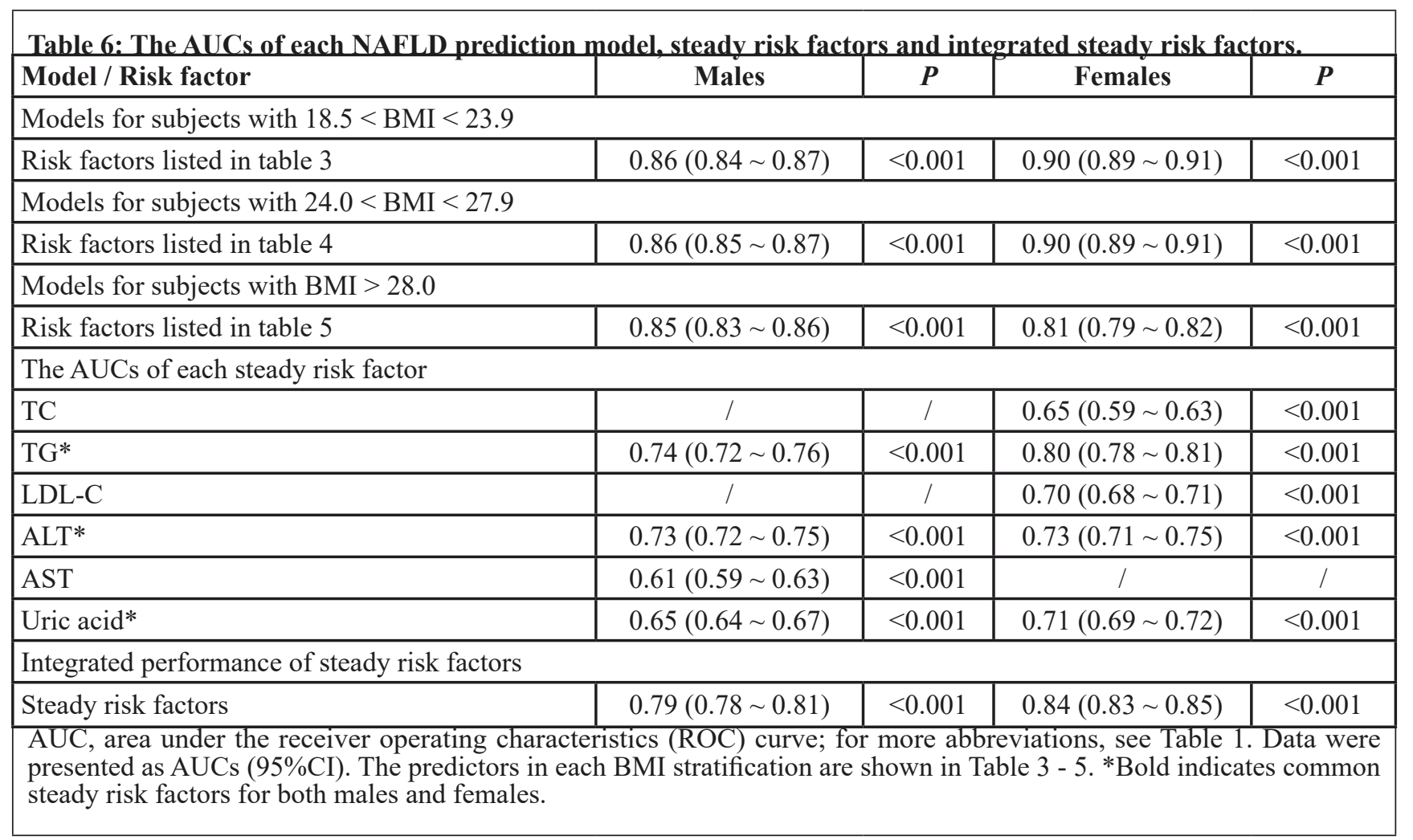

of NAFLD from general processes is problematic [23]. In addition, other biomarkers/panels for NASH have been described [23]. Age, sex, insulin resistance, systemic hypertension, TG, ALT, AST, GGT, AST/ALT ratio, $\alpha 2-$ macroglobulin, haptoglobin, apolipoprotein A1, total bilirubin, fasting blood glucose and fibrosis-4 were found to be associated with NASH and have been used in various diagnostic panels [23, 27, 28]. These studies advanced our knowledge of NAFLD and NASH and guided subsequent research. However, almost all of these studies were performed as shown in our Table 2, and did not take into consideration the possibility of altered risk factor compositions according to BMI; this might reduce the performance of diagnostic panels for discrimination of NAFLD. To evaluate our hypothesis, we recruited 8817 subjects. The subjects were divided into underweight, normal weight, overweight and obese groups, and the risk factor compositions and their association patterns were evaluated according to gender. First, in subjects with BMI $<18.5 \mathrm{~kg} / \mathrm{m}^{2}$, only two NAFLD patients were found among 445 subjects. The percentage of NAFLD increased sharply from $0.4 \%$ in this group to $81.9 \%$ in the obese group, indicating that BMI is associated tightly with morbidity and indicated the necessity of BMI stratification in the following analysis. Second, the NAFLD risk factor composition are affected markedly by the BMI of the subjects in both males and females, which suggests a pathophysiological dynamic characteristic of the process of NAFLD development and emphasises the importance of BMI stratification in NAFLD studies. Third, TG, ALT,
AST and uric acid are steady risk factors for NAFLD in males; and TC, TG, LDL-C, ALT and uric acid are steady risk factors for NAFLD in females; these risk factors displayed high performance for discrimination of NAFLD. Fourth, TG, ALT and uric acid are the common risk factors in both males and females with high performance in discrimination of NAFLD. Fifth, although BMI was used to group the subjects, it remained a risk factor for NAFLD in two-thirds of the stratifications (the normal weight, overweight and obese groups) in both males and females, with the highest AUC value. Sixth, urea nitrogen was not a risk factor in males or females, regardless of BMI stratification. Finally, the BMI, age, male ratio, systolic pressure, diastolic pressure, serum levels of TC, TG, LDL-C, glucose, ALT, AST, urea nitrogen, uric acid and creatinine were higher in groups with higher BMI, whereas HDL-C levels decreased with BMI.

The liver is the principle location of amino acid synthesis, protein degradation, carbohydrate metabolism, cholesterol synthesis, the production of triglycerides and the bulk of lipoprotein synthesis, as well as several other regulatory and growth factors [29-31]. Thus it is understandable that liver diseases correlate with metabolic disorders [31]. Regarding the liver lipid synthesis indexes, TG exhibited the greatest increase, from $0.8 \pm 0.35 \mathrm{mM}$ in the underweight group to $2.0 \pm 1.5 \mathrm{mM}$ in obese group, and was a steady risk factor for NAFLD in both males and females. By contrast, the elevating rate of $\mathrm{TC}$ was relatively slower and TC was a steady risk factor for NAFLD in females only. The above evidence suggests 
different roles for $\mathrm{TG}$ and $\mathrm{TC}$ in the pathogenesis of NAFLD in males and females. Uric acid and creatinine are indexes of metabolic and kidney function. They increase with increasing BMI, and intriguingly, are also risk factors for NAFLD. Hyperuricemia is one of the most common metabolic disorders in clinical practice, a large number of cross-sectional and several prospective studies show that hyperuricemia is associated with increased prevalence, incidence and disease severity of NAFLD [32-34]. In this report, uric acid is a common risk factor for both males and females, which consistent with previous reports [32-34]. The serum glucose level was also higher in subjects with higher BMI, although the mean did not exceed the upper limit of normal. Serum glucose level is an independent risk factor for NAFLD in both males and females who are normal weight or overweight; interestingly, serum glucose is not a risk factor for NAFLD in males and females with obesity. Systolic and diastolic blood pressures increased gradually with increasing BMI, and the average systolic pressure exceeded the upper limit of normal in the overweight and obese groups. Furthermore, systolic and diastolic pressures were independent risk factors for NAFLD in males in certain BMI stratifications. Intriguingly, systolic pressure was not a risk factor for NAFLD in females, but diastolic pressure was a risk factor for NAFLD in females with normal body weight. Our data suggest that the pattern of association between blood pressure and NAFLD varies according to gender and BMI and between systolic and diastolic pressure.

Levels of ALT and AST, indicators of liver injury and repair [19-21], increased with increasing BMI. Although the elevated ALT and AST levels did not exceed the normal ranges in our population, ALT was a common risk factor for NAFLD in both males and females regardless of BMI stratification; AST was a steady risk factor for NAFLD in males regardless of BMI stratification; AST was also an independent risk factor for NAFLD in females, with the exception of obese females. The above findings suggest that steatosis and/or mild inflammation of NAFLD lead to subclinical hepatocytes damage, which characterized by the stable association between elevated ALT and AST levels and NAFLD. It has been considered that AST/ALT $>0.8$ indicates $\mathrm{NASH}$ and AST/ALT $>1.0$ indicates progressive hepatic fibrosis [21,35]. Intriguingly, the overall average AST/ ALT ratios (without gender stratification) were $>1.0$ and increased from $1.0 \pm 0.4$ in the obese group to $1.7 \pm 0.6$ in the underweight group. Again, we emphasise that our subjects were recruited from the general adult population and underwent physical examinations; individuals with significant alcohol consumption ( $>20$ g per day), medications, parenteral nutrition, Wilson's disease, severe malnutrition, haemochromatosis, autoimmune liver disease, and/or chronic hepatitis were excluded. The AST and ALT levels of all participants were in the normal ranges. These factors might explain the $>1.0$ average AST/
ALT ratios in each group. Because existing noninvasive $\mathrm{NASH}$ evaluation strategies using the AST/ALT ratio are derived mainly from patients with chronic hepatitis and have not been validated in different ethnicities or at multiple centres [23], we then had given up the analysis of the risk factors for AST/ALT $\geq 1.0$ or 0.8 . Thus, although we could not distinguish NASH subjects from NAFLD population pathologically, the normal liver function and the exclusion criteria of participants made the subjects involved were mainly NAFLD but not NASH. Because NASH develops from NAFLD and weight loss is important for all patients with NAFLD [4], control and prevention of NAFLD is a cost-effective measure to reduce the morbidity of NASH. Our findings indicate a gender- and BMI-specific risk factor composition, which will facilitate individualised treatment strategies.

The limitations of this report are: 1) we did not include all known risk factors for NAFLD and hence we could also not provide a comprehensive assessment of the correlations between risk factors and NAFLD; 2) we did not categorise the pathological stages of NAFLD, so the associations of these factors with inflammation and NASH are unknown; 3) we did not perform liver biopsy, therefore our NAFLD diagnosis was based on a noninvasive approach.

\section{MATERIALS AND METHODS}

\section{Participants}

The study was approved by the Review Board of Shanghai Jiao Tong University Affiliated Sixth People's Hospital and conducted according to the principles of the World Medical Association Declaration of Helsinki. All subjects provided written informed consent prior to participating in this study. The participants were simultaneously informed of their right to repeal consent by them or their kin, caretakers, or guardians.

To investigate the risk factors associated with NAFLD in the general adult population, subjects that underwent routine physical examination in our hospital were recruited sequentially from June 2014 to August 2014. All participants underwent physical examinations and anthropometry, blood biochemistry and abdominal ultrasound evaluations. The diagnosis of NAFLD was established by following the Hepatic Steatosis Ultrasound Images Assessment Procedures Manual [36]. Briefly, the following ultrasonographic features were assessed, scored, and recorded: liver to kidney contrast; parenchymal brightness (i.e., hyperechogenic liver tissue with fine, tightly packed echoes); deep beam attenuation (i.e., the decreased ability of the ultrasound beam to penetrate the liver tissue causing posterior darkness and loss of definition of the diaphragm); bright vessel walls (i.e., 
the presence of bright walls of small intrahepatic vessels including the portal or hepatic veins); and gallbladder wall definition. The final diagnosis was based on the presence or absence of these five criteria. Individuals with significant alcohol consumption $(>20 \mathrm{~g}$ per day), medications, parenteral nutrition, Wilson's disease, severe malnutrition, haemochromatosis, autoimmune liver disease and/or chronic viral hepatitis were excluded. In addition, since the participants were general adult subjects underwent physical examinations, subjects with abnormal liver function were not included in this study. Finally, 8817 out of 10109 patients were included in this study.

\section{Data collection}

A questionnaire was self-administered by all participants; the items included age, sex, ethnicity, medical history, family history, smoking history and alcohol abuse. Anthropometric data were measured using standard methods with the participants dressed in lightweight clothing and with bare feet [37]. The BMI was calculated as body weight divided by height squared $\left(\mathrm{kg} / \mathrm{m}^{2}\right)$ [37]. According to the categories recommended by the Chinese Ministry of Health, BMI $<18.5$ refers to underweight, $18.5<\mathrm{BMI}<23.9$ refers to healthy weight, $24.0<\mathrm{BMI}<27.9$ refers to overweight, and BMI $>28.0$ refers to obese. Blood pressure was measured using a mercury sphygmomanometer in a seated position after a 5-min rest and was recorded as the mean of two different measurements taken at 1-min intervals. A fasting blood sample was collected from each participant via the antecubital vein in the morning. Glucose; serum lipids including total cholesterol (TC), triglycerides (TG), low-density lipoprotein-C (LDL-C), high-density lipoprotein-C (HDL-C), apolipoprotein (apo)A, apoE, apoB and lipoprotein (a) $\mathrm{Lp}(\mathrm{a})$; indicators of liver function including alanine aminotransferase (ALT) and aspartate aminotransferase (AST); indicators of kidney function, including urea nitrogen, uric acid and creatinine were measured in the hospital laboratory according to routine procedures.

\section{Statistical analysis}

The distribution tendency of BMI, age, systolic pressure, diastolic pressure, TC, TG, HDL-C, LDL-C, apoA, apoB, apoE, Lp(a), glucose, ALT, AST, urea nitrogen, uric acid and creatinine were examined using nonparametric tests. The correlation pattern between all variables and NAFLD was explored primarily using scatter and P-P diagrams. Pearson's correlation coefficients between each continuous variable were calculated prior to further statistical analysis. Collinearity diagnostics of all variables were also performed before further statistical analysis. After integrating the clinical significance,
Pearson's correlation coefficients and collinearity diagnostics, TC, TG, HDL-C and LDL-C were selected to represent the serum lipid profile.

To identify the risk factor panels associated with NAFLD in subjects with different BMI values, the subjects were placed into underweight (BMI $<18.5)$, normal $(18.5$ $<$ BMI < 23.9), overweight $(24.0<\mathrm{BMI}<27.9)$ and obese $(\mathrm{BMI}>28.0)$ groups according to the definition recommended by the Chinese Ministry of Health. Binary logistic regression was performed in each BMI group, and similar selected variable sets were introduced into the starting model and then eliminated manually using the backward step-by-step approach, depending on the largest $p$-value. All items showing statistical significance at $p<0.05$ were retained in the final equation. To evaluate the performance of each NAFLD prediction model, steady risk factors and integrated steady risk factors for males and females in the discrimination of NAFLD, area under the receiver operating characteristic (ROC) curve (AUC) analyses were also performed. All analyses were performed using SPSS for Windows (version 18).

\section{ACKNOWLEDGMENT}

This study was supported by the cross research foundation for medicine and engineering, Shanghai Jiao Tong University (YG2013MS49).

\section{CONFLICTS OF INTEREST}

The authors have declared that no conflict of interest.

\section{REFERENCES}

1. Kleiner DE, Brunt EM, Van Natta M, Behling C, Contos MJ, Cummings OW, Ferrell LD, Liu YC, Torbenson MS, Unalp-Arida A, Yeh M, McCullough AJ, Sanyal AJ; Nonalcoholic Steatohepatitis Clinical Research Network. Design and validation of a histological scoring system for nonalcoholic fatty liver disease. Hepatology. 2005; 41: 1313-1321.

2. Ratziu V, Bellentani S, Cortez-Pinto H, Day C, Marchesini G. A position statement on NAFLD/NASH based on the EASL 2009 special conference. J Hepatol. 2010; 53: 372384.

3. Chalasani N, Younossi Z, Lavine JE, Diehl AM, Brunt EM, Cusi K, Charlton M, Sanyal AJ. The diagnosis and management of non-alcoholic fatty liver disease: practice Guideline by the American Association for the Study of Liver Diseases, American College of Gastroenterology, and the American Gastroenterological Association. Hepatology. 2012; 55: 2005-2023.

4. Rinella ME. Nonalcoholic fatty liver disease: a systematic review. JAMA. 2015; 313: 2263-2273. 
5. Ludwig J, Viggiano TR, McGill DB, Oh BJ. Nonalcoholic steatohepatitis: Mayo Clinic experiences with a hitherto unnamed disease. Mayo Clin Proc. 1980; 55: 434-438.

6. Singh S, Allen AM, Wang Z, Prokop LJ, Murad MH, Loomba R. Fibrosis progression in nonalcoholic fatty liver vs nonalcoholic steatohepatitis: a systematic review and meta-analysis of paired-biopsy studies. Clin Gastroenterol Hepatol. 2015; 13: 643-654, e9.

7. Pais R, Charlotte F, Fedchuk L, Bedossa P, Lebray P, Poynard T, Ratziu V; LIDO Study Group. A systematic review of follow-up biopsies reveals disease progression in patients with non-alcoholic fatty liver. J Hepatol. 2013; 59: 550-556.

8. Williams CD, Stengel J, Asike MI, Torres DM, Shaw J, Contreras M, Landt CL, Harrison SA. Prevalence of nonalcoholic fatty liver disease and nonalcoholic steatohepatitis among a largely middle-aged population utilizing ultrasound and liver biopsy: a prospective study. Gastroenterology. 2011; 140: 124-131.

9. Chalasani N, Younossi Z, Lavine JE, Diehl AM, Brunt EM, Cusi K, Charlton M, Sanyal AJ. The diagnosis and management of non-alcoholic fatty liver disease: practice guideline by the American Gastroenterological Association, American Association for the Study of Liver Diseases, and American College of Gastroenterology. Gastroenterology. 2012; 142: 1592-1609.

10. Weiß J, Rau M, Geier A. Non-alcoholic fatty liver disease: epidemiology, clinical course, investigation, and treatment. Dtsch Arztebl Int. 2014; 111: 447-452.

11. Younossi ZM, Stepanova M, Afendy M, Fang Y, Younossi Y, Mir H, Srishord M. Changes in the prevalence of the most common causes of chronic liver diseases in the United States from 1988 to 2008. Clin Gastroenterol Hepatol. 2011; 9: 524-530.

12. Kim NH, Park J, Kim SH, Kim YH, Kim DH, Cho GY, Baik I, Lim HE, Kim EJ, Na JO, Lee JB, Lee SK, Shin C. Non-alcoholic fatty liver disease, metabolic syndrome and subclinical cardiovascular changes in the general population. Heart. 2014; 100: 938-943.

13. Alshehri AM. Metabolic syndrome and cardiovascular risk. J Family Community Med. 2010; 17: 73-78.

14. Anstee QM, Targher G, Day CP. Progression of NAFLD to diabetes mellitus, cardiovascular disease or cirrhosis. Nat Rev Gastroenterol Hepatol. 2013; 10: 330-344.

15. Hassan K, Bhalla V, El Regal ME, A-Kader HH. Nonalcoholic fatty liver disease: a comprehensive review of a growing epidemic. World J Gastroenterol. 2014; 20: 12082-12101.

16. Poynard T, Ratziu V, Naveau S, Thabut D, Charlotte F, Messous D, Capron D, Abella A, Massard J, Ngo Y, Munteanu M, Mercadier A, Manns M, et al. The diagnostic value of biomarkers (SteatoTest) for the prediction of liver steatosis. Comp Hepatol. 2005;4:10.

17. Bellentani S, Miglioli L, Masutti F, Passalacqua M,
Castiglione A, Tiribelli C. The Fatty Liver Index: a simple and accurate predictor of hepatic steatosis in the general population. BMC Gastroenterol. 2006; 6: 33.

18. Bedogni G, Kahn HS, Bellentani S, Tiribelli C. A simple index of lipid overaccumulation is a good marker of liver steatosis. BMC Gastroenterol. 2010; 10: 98.

19. Kotronen, A, Peltonen M, Hakkarainen A, Sevastianova K, Bergholm R, Johansson LM, Lundbom N, Rissanen A, Ridderstråle M, Groop L, Orho-Melander M, Yki-Järvinen H. Prediction of non-alcoholic fatty liver disease and liver fat using metabolic and genetic factors. Gastroenterology. 2009; 137: 865-872.

20. Lee JH, Kim D, Kim HJ, Lee CH, Yang JI, Kim W, Kim YJ, Yoon JH, Cho SH, Sung MW, Lee HS. Hepatic steatosis index: a simple screening tool reflecting nonalcoholic fatty liver disease. Dig Liver Dis. 2010; 42: 503-508.

21. Castera L, Vilgrain V, Angulo P. Noninvasive evaluation of NAFLD. Nat Rev Gastroenterol Hepatol. 2013; 10: 666675.

22. Sattar N, Forrest E, Preiss D. Non-alcoholic fatty liver disease. BMJ. 2014; 349: g4596.

23. Sanal, M.G. Biomarkers in nonalcoholic fatty liver diseasethe emperor has no clothes? World J Gastroenterol. 2015; 21: 3223-3231.

24. Wieckowska A, Papouchado BG, Li Z, Lopez R, Zein NN, Feldstein AE. Increased hepatic and circulating interleukin-6 levels in human nonalcoholic steatohepatitis. Am J Gastroenterol. 2008; 103:1372-1379.

25. Feldstein AE, Lopez R, Tamimi TA, Yerian L, Chung YM, Berk M, Zhang R, McIntyre TM, Hazen SL. Mass spectrometric profiling of oxidized lipid products in human nonalcoholic fatty liver disease and nonalcoholic steatohepatitis. J Lipid Res. 2010; 51: 3046-3054.

26. Cermelli S, Ruggieri A, Marrero JA, Ioannou GN, Beretta L. Circulating microRNAs in patients with chronic hepatitis $\mathrm{C}$ and non-alcoholic fatty liver disease. PLoS One. 2011; 6: e23937.

27. Dixon JB, Bhathal PS, O'Brien PE. Nonalcoholic fatty liver disease: predictors of nonalcoholic steatohepatitis and liver fibrosis in the severely obese. Gastroenterology. 2001; 121 : 91-100.

28. Palekar NA, Naus R, Larson SP, Ward J, Harrison SA. Clinical model for distinguishing nonalcoholic steatohepatitis from simple steatosis in patients with nonalcoholic fatty liver disease. Liver Int. 2006; 26: 151156.

29. Cohen DE, Fisher EA. Lipoprotein metabolism, dyslipidemia, and nonalcoholic fatty liver disease. Semin Liver Dis. 2013; 33: 380-388.

30. Cooper AD. Role of the liver in the degradation of lipoproteins. Gastroenterology. 1985; 88: 192-205.

31. Matos C, Porayko MK, Francisco-Ziller N, DiCecco S. Nutrition and chronic liver disease. J Clin Gastroenterol. 2002; 35: 391-397. 
32. Xu C. Hyperuricemia and nonalcoholic fatty liver disease: from bedside to bench and back. Hepatol Int. 2016;10:28693.

33. Li Y, Xu C, Yu C, Xu L, Miao M. Association of serum uric acid level with non-alcoholic fatty liver disease: a crosssectional study. J Hepatol. 2009;50:1029-34.

34. Xu C, Yu C, Xu L, Miao M, Li Y. High serum uric acid increases the risk for nonalcoholic Fatty liver disease: a prospective observational study. PLoS One. 2010;5:e11578.

35. Sheth SG, Flamm SL, Gordon FD, Chopra S. AST/ALT ratio predicts cirrhosis in patients with chronic hepatitis $\mathrm{C}$ virus infection. Am J Gastroenterol. 1998; 93: 44-48.

36. National Health and Nutrition Examination Survey (NHANES) III. Hepatic Steatosis Ultrasound Images Assessment Procedures Manual (2010) Available at : http://www.cdc.gov/nchs/data/nhanes/nhanes3/Hepatic Steatosis_Ultrasound_Procedures_Manual.pdf. (Accessed: 4th April 2016)
37. World Health Organization, Waist circumference and waist-hip ratio, Report of a WHO expert consultation. (2008) Available at: http://www.who.int/nutrition/ publications/obesity/WHO_report_waistcircumference and waisthip_ratio/en/. (Accessed: 4th April 2016) 